\title{
Command Information System Structure Complexity Analysis Method
}

\author{
Yuxiao $\mathrm{Kou}^{1 *}$, Aimin $\mathrm{Luo}^{1}$, Yi Mao ${ }^{2}$, Xiaoxue Zhang1, 2, Zhen Shu ${ }^{1}$ \\ ${ }^{1}$ Science and Technology on Information Systems Engineering Laboratory, National University of Defense \\ Technology, Changsha, Hunan, 410073, P.R.China. \\ ${ }^{2}$ State Key Laboratory of Air Traffic Management System and Technology, Nanjing, 210007, China. \\ * Corresponding author. Tel.: +8615580838796; email: 1466030719@qq.com \\ Manuscript submitted May 4, 2019; accepted July 10, 2019. \\ doi: $10.17706 /$ jsw.14.8.370-379
}

\begin{abstract}
Based on the structural characteristics of command information system, the super-network model of the system is established. In the system, the four types of basic units with different functions are the supernetwork nodes. And the information flow motifs defined by the specific information flow structure are the edges of the super-network. Then, based on the entropy theory, the $x$ - information flow motif entropy, the $n$ - information flow motif entropy and the $n(t)$ information flow motif entropy are defined. The three levels of entropy are superimposed as an index to measure the structural complexity of the C ${ }^{4}$ ISR system. Verification is carried out through a case of a joint air defense system in a certain area.
\end{abstract}

Key words: Command information system, super-network, information flow motif entropy.

\section{Introduction}

As the most fundamental material basis and support of information warfare, command information system plays an irreplaceable role in modern warfare. However, the complexity of the system structure not only increases the cost of system research and construction, but also may cause unexpected changes in the system. Quantitative evaluation of the complexity of C4ISR system can help to control system complexity and optimize network structure in the design process.

Existing system complexity research can be broadly divided into two categories. One is based on the theory of complex network, using the concepts of graph theory to evaluate complexity. For example, Huaibin Qin $e t$ al. [1] abstracted the software system into a directed complex network model, and evaluated the complexity of the software system through some complex network characteristic parameters such as cluster coefficient. The other is related to entropy theory. For example, Meng Cai et al. [2] defined a new network structure entropy by taking the difference of "point" and "edge" into consideration, and carried out theoretical analysis and simulation experiment on the structure entropy of regular network, random network and scale-free network. Yuying Wu et al. [3] put forward the concept of network structure entropy based on motif theory, and measured the structure complexity of a cooperative network as a case.

Due to the heterogeneity of nodes and the multiplicity of links, the command information system is essentially a heterogeneous multi-edge network, and the existing methods are not suitable for the research of its structural complexity.

Hypernetwork theory is suitable for modeling and analysis of heterogeneous multilateral networks. 
Therefore, this paper models the C4ISR system using the super-network theory. And for the information flow structure unique to the command information system, the entropy of the information flow motif at three levels are defined based on the motif theory. Then the different levels of entropy are superimposed as an index to measure the structural complexity of the C4ISR system.

\section{Super-network Theory}

The concept of hypernetwork originated from the research on traffic network, and there is still no accepted definition now. Nagurney, an American scientist, believes that hypernetwork is "above and beyond existing networks"[4]. However, hypernetwork can be defined from different perspectives. For example, a hypergraph can be used to define the hypernetwork.

Supposing $V=\left\{v_{1}, v_{2}, \ldots, v_{n}\right\}$ is a finite set, if

$$
\begin{aligned}
& e_{i} \neq \varnothing(i=1,2, . . m) \\
& \bigcup_{i=1}^{m} e_{i}=V
\end{aligned}
$$

Then a binary relation $H=(V, E)$ is called a hypergraph. The elements $v_{1}, v_{2}, \ldots, v_{n}$ of $V$ are the vertices of the hypergraph. $E=\left\{e_{1}, e_{2}, \ldots, e_{m}\right\}$ is the edge set of the hypergraph, and $e_{i}=\left\{v_{i 1}, v_{i 2}, \ldots, v_{i j}\right\}$ $(i=1,2, . . m)$ are the edges.

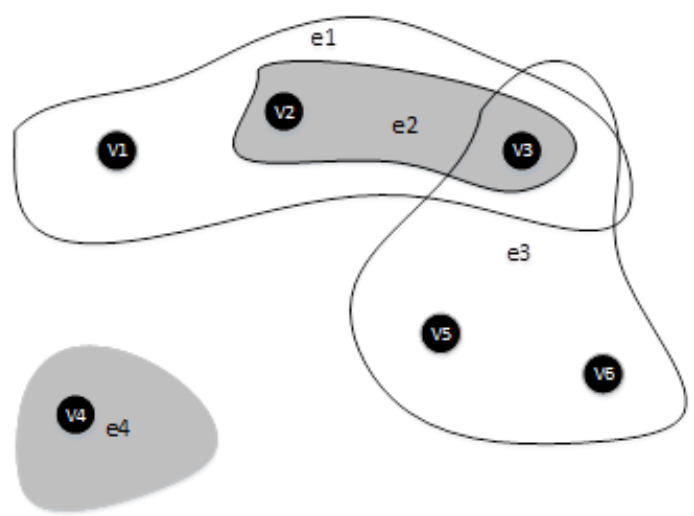

Fig. 1. Hypergraph Sample

As shown in Fig. 1, $V=\left\{v_{1}, v_{2}, v_{3}, v_{4}, v_{5}, v_{6}\right\}, E=\left\{e_{1}=\left\{v_{1}, v_{2}, v_{3}\right\}, e_{2}=\left\{v_{2}, v_{3}\right\}, e_{3}=\left\{v_{3}, v_{5}, v_{6}\right\}, e_{4}=\left\{v_{4}\right\}\right\}$. Then the command information system is modeled.

\section{Super-network Model of Command Information System}

In the structure of the command information system, both of the functions of the nodes and the types of information exchanged between the nodes are different. In addition, the process of executing tasks in the system follows the OODA model, that is, observe $\rightarrow$ orient $\rightarrow$ decide $\rightarrow$ act. Therefore, the command information system has three distinct characteristics: heterogeneous nodes, multiple links and military functional characteristics. Since the super-network theory is mainly applied to modeling heterogeneous multi-edge complex system which emphasizes the overall function, it is reasonable to use the super-network theory to model and analyze the structure of the command information system.

\subsection{Four Basic System Unit Models}


According to the functions and properties of nodes in the command information system, four basic system unit models are proposed. Table 1 gives the symbolic representation and function description of the unit models.

Table 1. Types of Command Information System Nodes

\begin{tabular}{|l|l|l|l|}
\hline Nodes Types & Symbol & Function & Instance \\
\hline Intelligence Acquisition & $O$ & Collect intelligence & Radar \\
\hline Intelligence Process & $P$ & Processing intelligence & Intelligence Integration Center \\
\hline Decision Making & $D$ & Make decisions based on situation & Command Center \\
\hline Response and Execute & $A$ & Execute the orders from the decision node & Weapon Platform \\
\hline
\end{tabular}

\subsection{Three Information Interaction Relationship Models}

The unit relationship models established in this paper are information relationship models. The information relationship describes the information exchanging between the system units, but it can't describe how the information exchange is realized, such as what technologies are adopted, what links are adopted and what communication nodes are passed through. Information relations are represented by directed edges, and the direction of the edges indicates the flow of information. The type of information relationship is closely related to the type of information content transmitted in the system, which can be divided into three categories:

1) Intelligence Information: the original information obtained through detection or monitoring equip ment and the intelligence information after the fusion of intelligence processing nodes.

2) Command and Control Information: the information generated by the command and control units to control the subordinate troops or weapons, including the command, plan and target.

3) Status Information: information generated by various system units to report the status of themselves and the surrounding environment.

According to the classification of information content, information relations can be divided into three categories. And each category corresponds to multiple instances, as shown in the following table:

Table 2. Information Interaction Relationship of Command Information System

\begin{tabular}{|l|l|}
\hline Type of Information Relationship & Relationship Instance \\
\hline Intelligence Guarantee and Sharing & $O \rightarrow A, O \rightarrow P, O \rightarrow D, P \rightarrow A, P \rightarrow D, P \rightarrow P$ \\
\hline Command and Control & $D \rightarrow A, D \rightarrow P, P \rightarrow O, D \rightarrow D, P \rightarrow P$ \\
\hline State Synchronization and Feedback & $A \rightarrow D, D \rightarrow A, P \rightarrow D, O \rightarrow P, P \rightarrow O, D \rightarrow D, P \rightarrow P$, \\
& $A \rightarrow A, O \rightarrow O$ \\
\hline
\end{tabular}

1) Intelligence Guarantee and Sharing Relationship: the information interaction relation between the system units that guarantee or share information and the system units that process or use information. This relationship mainly exchanges intelligence information. 
2) Information Command and Control Relationship: the relationship between command units and be commanded units, or between control units and be controlled units. This relationship mainly exchanges command and control information.

3) State synchronization and Feedback Relationship: the relation that the system units feedback the relevant state information to other system units. This relationship primarily exchanges state information.

\subsection{Information Flow Motif}

The network motif is the pattern of interconnection in the network, and the occurrence of this pattern in the complex network is significantly higher than that in the random network [5]. And the information flow mode refers to the information action mode of generating, processing and using information that occurs repeatedly in the ${ }^{4}$ ISR system structure [6]. Since the CIISR system performs tasks following the OODA model, the information flows presented as $O \rightarrow P \rightarrow D \rightarrow A$ style would appear in large numbers in the actual network structure. Therefore, in the command information system network, the OODA connection mode is the information flow motif of the network.

According to the three types of information and three types of information interaction relationship in the ${ }^{C}{ }^{4}$ ISR system, there are three basic information flow motifs in the system structure. Table 2 gives the detailed description.

Table 3. Information Flow Motif Table of C4ISR System

\begin{tabular}{|c|c|c|}
\hline $\begin{array}{l}\text { Type of } \\
\text { information flow } \\
\text { motif }\end{array}$ & $\begin{array}{l}\text { Pattern of } \\
\text { Information flow } \\
\text { motif }\end{array}$ & Description \\
\hline \multirow{2}{*}{$\begin{array}{l}\text { Intelligence } \\
\text { information flow } \\
\text { (IFlow) }\end{array}$} & $O \rightarrow P^{*} \rightarrow A$ & $\begin{array}{l}\text { The intelligence acquisition unit generates information and sends it to } \\
\text { the execution unit through the processing unit or directly to the } \\
\text { execution unit. }\end{array}$ \\
\hline & $O \rightarrow P^{*} \rightarrow D$ & $\begin{array}{l}\text { The intelligence acquisition unit generates information and sends it to } \\
\text { the decision unit through the processing unit or directly to the decision } \\
\text { unit. }\end{array}$ \\
\hline \multirow{3}{*}{$\begin{array}{l}\text { Command and control } \\
\text { information flow } \\
(\mathrm{C} 2 \text { Flow })\end{array}$} & $D^{+} \rightarrow A$ & The decision unit sends the command to the execution unit step by step. \\
\hline & $P^{+} \rightarrow O$ & $\begin{array}{l}\text { The intelligence processing unit sends the command to the detection } \\
\text { unit step by step. }\end{array}$ \\
\hline & $D \rightarrow P \rightarrow O$ & $\begin{array}{l}\text { The detector control command generated by the decision unit is sent to } \\
\text { the detection unit through the intelligence processing unit. }\end{array}$ \\
\hline \multirow{4}{*}{$\begin{array}{l}\text { Cooperation } \\
\text { information flow } \\
(\text { FFlow) }\end{array}$} & $D \rightarrow D^{*} \rightarrow D$ & Collaboration between decision units \\
\hline & $P \rightarrow P^{*} \rightarrow P$ & Collaboration between intelligence processing units \\
\hline & $A \rightarrow D^{*} \rightarrow A$ & Collaboration between execution units through decision units \\
\hline & $O \rightarrow P^{*} \rightarrow O$ & $\begin{array}{l}\text { Coordination between detection units through intelligence processing } \\
\text { units }\end{array}$ \\
\hline
\end{tabular}

\subsection{Super-network Model of C ${ }^{4}$ ISR System}

The super-network model $\mathrm{M}$ of command information system can be expressed as:

$$
M=\{O, P, D, A, \text { IFlow, C2Flow, FFLow }\}
$$


where $O$ represents the set of intelligence acquisition units set, $P$ represents the set of intelligence processing units, $D$ represents the set of decision units, $A$ represents the set of execution units, and $V=\{O, P, D, A\}$ is the node-set of the super-network model.

Information motifs IFlow, C2Flow, FFlow are super-edges of the super-network model, and $E=\{$ IFlow, C2Flow, FFlow $\}$ is the set of edges.

\section{Evaluation of System Structure Complexity Based on Motif Entropy Superposition}

There are three kinds of motif structures in the C4ISR system: intelligence information flow motif, command information flow motif and cooperation information flow motif. Therefore, the motif is taken as the basic element of the network to evaluate the structure complexity by calculating the motif entropy. The greater the diversity of the motifs, the greater the uncertainty of the network structure, that is, the more complex the structure.

\subsection{Definition of Information Flow Motif and Motif Entropy}

In the model of the command information system, due to the heterogeneity of nodes, the number of node types may be different in the motifs with the same number of nodes. Even if the motifs contain the same number of nodes as well as node types, there will be different types of combination. These all reflect the diversity of motifs, namely the complexity of the structure, which must be considered comprehensively. Therefore, the motifs are classified according to the total number of nodes and the number of node types contained in them. And the definitions are given below:

Definition 1: $n(t)$ information flow motif: the information flow motif whose total number of nodes is $n$ and the number of node types is $t$.

Definition 2: $n$ - information flow motif: the information flow motif whose total number of nodes is $n$ and the number of node types is uncertain.

Definition 3: $x$ - information flow motif: the information flow motif whose node types and the total number of nodes in the motif are uncertain.

From the perspective of information interaction, the definition of information flow motif entropy is given according to the three definitions of information flow motifs:

Definition 4: $n(t)$ information flow motif entropy $H^{n(t)}$ :

$$
H^{n(t)}=-\sum_{i=1}^{S_{n(t)}} p_{i}^{n(t)} \log p_{i}^{n(t)}
$$

where $S_{n(t)}$ represents the number of types of $n(t)$ motifs in the network, and $p_{i}^{n(t)}$ is the ratio of the number of $n(t)$ motifs of type $i$ to the total number of $n(t)$ motifs. For example, the 3(2) motifs in a command information system has three types: $D \rightarrow D \rightarrow A, A \rightarrow D \rightarrow A$ and $P \rightarrow P \rightarrow O$, then $S_{3(2)}=3$. And the proportion of each type is $1 / 3$.

Definition 5: $n$-information flow motif entropy $H^{n-}$ :

$$
H^{n-}=-\sum_{i=1}^{S_{n-}} p_{i}^{n-} \log p_{i}^{n-}
$$

where $S_{n-}$ represents the number of types of $n$ - motifs whose number of node types $t$ are different, 
and $p_{i}^{n-}$ is the ratio of the number of $n-$ motifs of type $i$ to the total number of $n$ - motifs. For example, there are 4(2), 4(3) and 4(4) motifs in a network for 4- motifs. Then the number of types of 4- motifs is 3 , that is $S_{4-}=3$.

Definition 6: The entropy of the global information flow motif in the network $H^{x-}$ :

$$
H^{x-}=-\sum_{i=1}^{S_{x}} p_{i}^{x} \log p_{i}^{x}
$$

where $S_{x}$ represents the number of motif types in which the total number of nodes $n$ contained in the motifs are different, and $p_{i}^{x}$ is the ratio of the number of motifs of type $i$ to the number of all motifs in the network. For example, all the motifs in a command information system are divided into 3-, 4-, 5- and 6- motifs according to the number of nodes contained. Then there are 4 types of motifs with different total nodes, that is $S_{x}=4$.

Therefore, the information flow motifs in the network are divided into 3 levels. The bottom level is the $n(t)$ motif whose total number of nodes and the number of node types are fixed. And the $n(t)$ motif entropy measures the number of different combinations of node types in $n(t)$ motifs. The second level is the $n$ - motif whose total number of nodes $n$ is fixed. When calculating the $n$ - motif entropy, it evaluates the difference in the total number of node types contained in $n-$ motifs. The top level is the $x-$ motif whose number of nodes is uncertain and its motif entropy measures the difference in the number of nodes of all motifs in the network.

In the network, when the $n-$ motif entropy and the $x$ - motif entropy are constant, higher $n(t)$ motif entropy means more types of $n(t)$ motifs. And the network structure is more complex. When the $n(t)$ motif entropy and the $x$ - motif entropy are constant, if the $n$ - motif entropy is higher, the distribution of $n$ - motifs is more disordered, and the network structure is more complicated. Similarly, when the $n(t)$ motif entropy and the $n$ - motif entropy are constant, if the $x$ - motif entropy is higher, the distribution of $x$ - motifs is more disordered, and the network structure is more complex. Therefore, the complexity of the logical structure of the system is an increasing function about the three types of motif entropy.

\subsection{Evaluation Index based on Motif Entropy Superposition}

When using motif entropy to measure network complexity, the key is to fully represent the diversity of motifs. Motif entropy is an index to describe the diversity of motifs, and different levels of motif entropy describes different levels of motifs. In order to fully consider the diversity of all the motifs of the whole network, it is necessary to combine the $n(t)$ motif entropy, $n$ - motif entropy and $x$ - motif entropy, which are progressive in the level. At the same time, no matter which level of motif entropy increases, the index which evaluates the structure complexity will increase, so the index of complexity is defined as follows:

Definition 7: $n-$ motif complexity Complexity $^{n-}$ :

$$
\text { Complexity }^{n-}=\left(1+\sum_{i=1}^{S_{n-}} H^{n(t)}\right) H^{n-}
$$

Definition 8: global motif complexity Complexity ${ }^{\text {all }}$ : 


$$
\text { Complexity }^{\text {all }}=\left(1+\sum_{i=1}^{S_{x}} \text { Complexity }^{n-}\right) H^{x-}
$$

In the definition 7, the sum of all the $n(t)$ motif entropy corresponding to the $n$ - motif is used as the growth ratio of $H^{n-}$ to obtain the $n$ - motif complexity Complexity ${ }^{n-}$. If the value of $H^{n(t)}$ or $H^{n-}$ is larger, then the value of Complexity ${ }^{n-}$ will be larger.

Similarly, the sum of all the Complexity ${ }^{n-}$ is used as the growth ratio of $H^{x-}$ to obtain the global motif complexity Complexity ${ }^{\text {all }}$. If the value of Complexity $^{n-}$ or $H^{x-}$ is larger, then the value of Complexity ${ }^{\text {all }}$ will be larger.

In conclusion, Complexity ${ }^{\text {all }}$ increases with the increase of $H^{n(t)}, H^{n-}$ and $H^{x-}$, and it can comprehensively reflect the diversity of the motifs in the system from multiple levels.

The network motif is the basic unit of the network. And the greater the diversity of motifs, the more complex the network structure. Therefore, the global motif complexity Complexity ${ }^{\text {all }}$, which can reflect the diversity of motifs, also represents the complexity of network structure.

\section{Case Analysis}

By the detection of the radar brigade, a regional joint air defense combat mission is to intercept the enemy aircraft that invaded the airspace through joint action of air unit and ground brigades. The total number of nodes in the regional joint air defense command information system is 36 , including 10 command and control nodes, 6 intelligence processing nodes, 12 intelligence detection nodes and 8 execution nodes.

According to the regional joint air defense combat mission, two kinds of command information system structures are given. Fig. 2a shows the first structure, which is a typical treelike system structure. The second structure shown in Figure $1 \mathrm{~b}$ is based on the first structure, adding the intelligence and situation sharing relationship as well as the bypassing commanding relationship. And in Fig. $2 \mathrm{~b}$, the radar brigade unit also adds the intelligence integration function in its area of responsibility. Therefore, the second system has a flat structure.

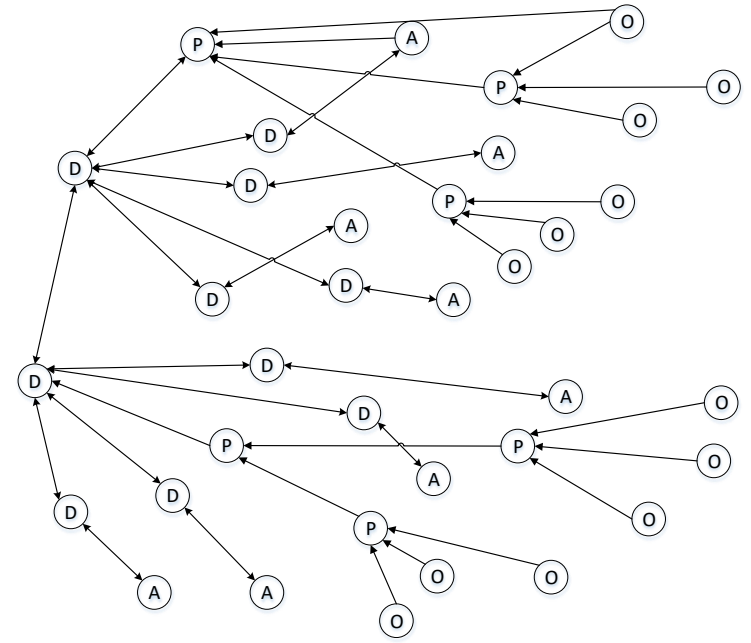

Fig. 2a. System structure 1.

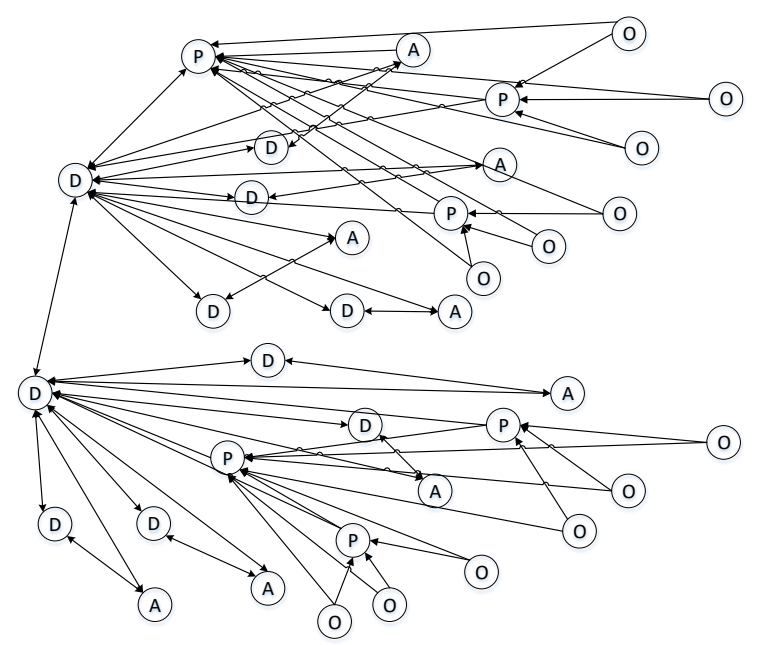

Fig. 2b. System structure 2.

\subsection{System Structural Modeling}


In order to build the super-network model of the system, it is necessary to find out all the information flow motifs in the network. In this paper, the information flow motif searching algorithm is adopted. The algorithm defines all the possible forms of the information flow motif in the network. Then the function is defined to find and count motifs according to their possible forms. Table 3 shows the number of information flow motifs in the two structures.

Table 4. The Number of Information Flow Modules in Structure 1 and 2

\begin{tabular}{|l|l|l|l|}
\hline $\begin{array}{l}\text { Type of } \\
\text { information flow motif }\end{array}$ & Pattern of Information flow motif & statistics of Structure1 & statistics of Structure2 \\
\hline \multirow{3}{*}{ IFlow } & $O \rightarrow P \rightarrow D$ & 1 & 24 \\
\cline { 2 - 4 } & $O \rightarrow P \rightarrow P \rightarrow D$ & 12 & 12 \\
\hline \multirow{3}{*}{ C2Flow } & $D \rightarrow A$ & 0 & 8 \\
\hline \multirow{4}{*}{ FFlow } & $D \rightarrow D \rightarrow A$ & 8 & 8 \\
\cline { 2 - 4 } & $A \rightarrow D \rightarrow D \rightarrow D \rightarrow A$ & 12 & 12 \\
\cline { 2 - 4 } & $A \rightarrow D \rightarrow A$ & 0 & 12 \\
\cline { 2 - 4 } & $A \rightarrow D \rightarrow D \rightarrow A$ & 0 & 24 \\
\hline
\end{tabular}

\subsection{Structural Complexity Analysis}

As shown in Figure 3a and Figure 3b, the information flow motifs of structure 1 and 2 are layered according to the $n(t)$ and $n$ - motifs to analyze the complexity of the logical structure of the system.

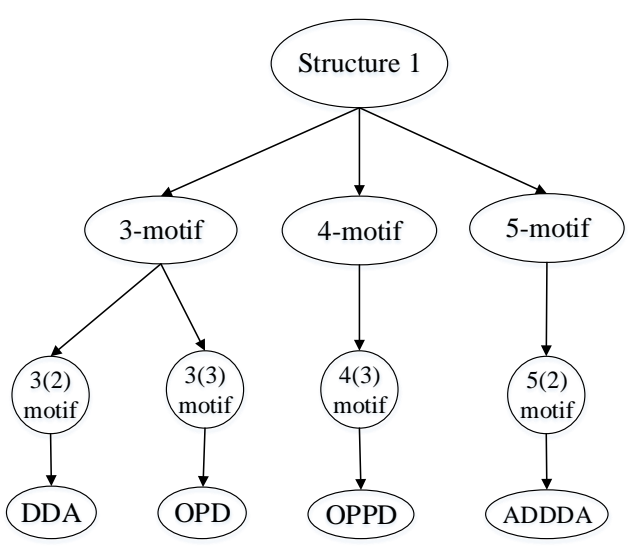

Fig. 3a. Motif Layering of Structure 1

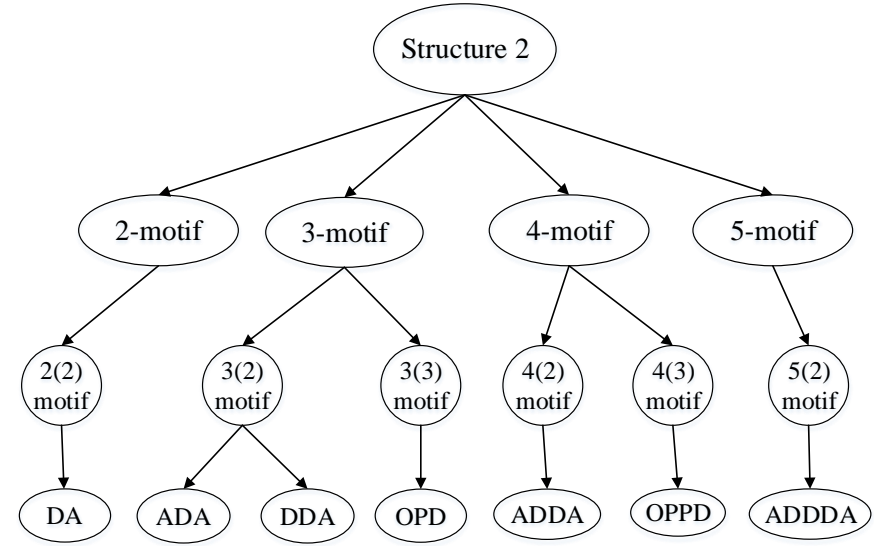

Fig. 3b. Motif Layering of Structure 2

Then the $n(t)$ motif entropy, $n$ - motif entropy and $x$ - motif entropy of structure 1 and 2 are calculated respectively. Table 5 and 6 show the results.

Table 5. $H^{n(t)}$ of Structure 1 and Structure 2

\begin{tabular}{|l|l|l|l|l|l|l|}
\hline & $H^{2(2)}$ & $H^{3(2)}$ & $H^{3(3)}$ & $H^{4(2)}$ & $H^{4(3)}$ & $H^{5(2)}$ \\
\hline structure 1 & $/$ & 0 & 0 & $/$ & 0 & 0 \\
\hline structure 2 & 0 & 0.29 & 0 & 0 & 0 & 0 \\
\hline
\end{tabular}


Table 6. $H^{n-}$ and $H^{x-}$ of Structure 1 and Structure 2

\begin{tabular}{|c|l|l|l|l|l|}
\hline & $H^{2-}$ & $H^{3-}$ & $H^{4-}$ & $H^{5-}$ & $H^{x-}$ \\
\hline structure 1 & $/$ & 0.15 & 0 & 0 & 0.47 \\
\hline structure 2 & 0 & 0.30 & 0.28 & 0 & 0.51 \\
\hline
\end{tabular}

From Table 5 and Table 6, structure 2 has more types of $n(t)$ motifs than structure 1 . And structure 2 also has more types of $n$ - motifs than structure 1. Due to the difference in the type of $n(t)$ motifs and $n-$ motifs, the $n$ - motif entropy and $x$ - motif entropy of structure 2 are both larger than that of structure 1. Therefore, in the three levels of the motif, the motif type of structure 2 is richer than that of structure 1 , and the entropy value is also larger.

According to the calculation of Complexity ${ }^{\text {all }}$, the Complexity ${ }^{\text {all }}$ of structure 1 is 0.54 and the Complexity $^{\text {all }}$ of structure 2 is 0.85 . Therefore, structure 1 is more complex than structure 2. Compared with structure 1, structure 2 adds the intelligence sharing relationship, the bypassing commanding relationship and the bypassing reporting relationship. And the information flow motifs in structure 2 are more diverse, so the conclusion that the complexity of structure 2 is greater than that of structure 1 is reasonable.

\section{Conclusion}

How to evaluate the complexity of system is of great significance to the research of C ${ }^{4}$ ISR system. In this paper, the structure of the command information system is modeled based on the super-network theory. Then the information flow motif entropy of three levels are defined based on the entropy theory and the concept of the motif. And the three levels of entropy are superimposed as an index to evaluate the structural complexity of the C4ISR system. This is only an exploration of the system complexity problem. The next step will be to consider the system complexity over time.

\section{Acknowledgment}

This study is supported by State Key Laboratory of air Traffic Management System and Technology (No.SKLATM201806).

\section{References}

[1] Qin, H. B., Li, D. L., Guo, L., et al. (2013). Complexity metrics method for software Architecture based on complex networks. Microelectronics and Computer, 30(2), 5-8.

[2] Cai, M., Du, H. F., et al. (2011). A new network structure entropy based node difference and edge difference. Acta Physica Sinica, 60(11), 110513 -1-110513-9.

[3] Wu, Y. Y., Tan, Y. J., \& He, X. J. (2015). The measurement of network structure complexity based on motify. Science and Technology Management Research, 7, 204-208.

[4] Nagurney, A., \& Dong, J. (2002). Supernetworks: Decision-Making for the Information Age. Cheltenham: Edward Elgar Publishing.

[5] Milo, R., Shen, O. S., Itzkovitz, S., et al. (2002). Network motifs: simple building blocks of complex networks. Science, 298, 824-827.

[6] Lan, Y. S., Mao, S. J., et al. (2015). Command Information System Structure Theory and Optimization Method. Beijing: National Defense Industry Press. 


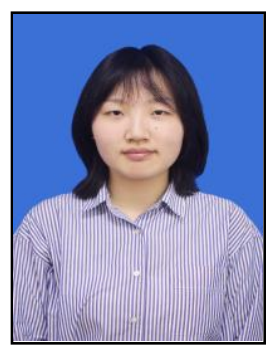

Yuxiao Kou was born in 1996. She received the bachelor of engineering degree in command information system engineering in 2017 from National University of Defense Technology, Changsha, China, where she is currently studying for a master's degree in management science and engineering. Her research is focused on analyzing and evaluating information systems.

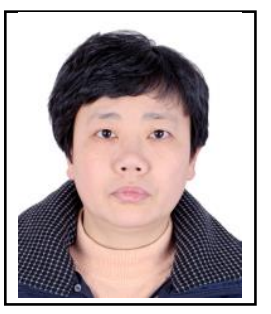

Aimin Luo was born in 1971. She received the M.Sc. degree and Ph.D. degree from National University of Defense Technology (NUDT), Changsha, P. R. China. She is a professor of management science and engineering. Her current research interests include requirement engineering, military information system, enterprise architecture design and analysis.

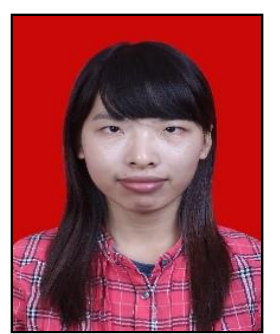

Xiaoxue Zhang was born in 1986. She received the Ph.D. degree in military science in 2014 from National University of Defense Technology (NUDT), Changsha, P. R.China. She is a lecturer of the School of System Engineering at NUDT. Her research interests are enterprise architecture design and analysis 